\title{
COMMUNICATIONS
}

\section{KERATO-CONJUNCTIVITIS SICCA ${ }^{*}$}

\author{
BY \\ A. STANWORTH \\ Department of Ophthalmology, University of Manchester
}

KERATO-CONJUNCTIVITIS sicca has been fairly prominent in the ophthalmic literature since Sjögren's classical series of papers (1933-1940), and has been reviewed recently by Henderson (1950). A further fifty cases are reviewed below.

\section{General Considerations}

The presumptive diagnosis was made on clinical grounds in the majority of cases, the signs and symptoms being those usually described-redness and discomfort, tenacious mucous discharge, and corneal erosions and filaments. Confirmation was obtained by the measurement of the lacrimal secretion by the filter-paper test (Schirmer, 1903) and in doubtful cases by staining with rose bengal. The relative value of these two tests is still in doubt, since neither gives a certain indication of the clinical severity of the condition. In addition, the normal values obtained by the filter-paper test are not known with. sufficient accuracy.

The usual criterion, using a piece of filter paper $5 \mathrm{~mm}$. broad, with $5 \mathrm{~mm}$. tucked over the lower lid, is that in five minutes a normal eye will produce enough moisture to wet the paper for a distance of more than $20 \mathrm{~mm}$. Less than $15 \mathrm{~mm}$. is suspicious of a dry eye, and less than $10 \mathrm{~mm}$. may be diagnostic. This method is not yet fully standardized; some, following Schirmer himself, place the filter paper at the outer canthus, some at the inner. The former is preferable, as there is less likelihood of a lake of lacrimal fluid at the outer than at the inner canthus, a condition which will give an initial rapid wetting of the paper and possibly a false reading, particularly if the lacrimal passages are for some reason occluded. Some observers

+ Based on a paper read before the North of England Ophthalmological Society, October 27, 1950.

* Received for publication February 20, 1951. 
also believe that a period shorter than five minutes is adequate; this is certainly the case in many patients, but one may misinterpret the doubtful case if the full period is not used. Quite commonly in a normal person, and especially in those with superficial ocular conditions not due to kerato-conjunctivitis sicca, the filter paper, even after five minutes, will show a progressive increase in the amount of wetting. In cases of kerato-conjunctivitis sicca, however, a steady state is reached within five minutes whereby the rate of addition of moisture from the conjunctival end is balanced by an equal rate of evaporation from the surface of the paper, and no further extension of the wet area is seen. It is therefore difficult to give an average figure for the normal. Particularly is this so if the so-called normal series includes many with some superficial irritation from an atmosphere which is none too clean, as is commonly the case in cities. A further point is that when the atmosphere is warm and dry the evaporation from the surface of the paper will be more rapid than under cool moist conditions. There is, indeed, a tendency for the wetting of the paper to be greater in winter than in summer in the milder cases of dry eye, but not in the severe ones (average of eight mild cases June to August, $8 \mathrm{~mm}$. Schirmer; average of the same cases November to February, $10 \mathrm{~mm}$.). To some extent this may be due to variations in the rate of evaporation, but is more probably due to a tendency for the eyes to be more irritable in winter than summer, the mild cases of dry eye responding with a further secretion of tears. In spite of this, the patient's symptoms are usually worse, the slightly increased supply failing to keep pace with the much increased demand. Possibly also there is some deficiency in the quality of the tears themselves; Ridley (1948), for example, found a deficiency in protein. This may explain those patients with typical keratoconjunctivitis sicca who have an apparently normal volume of secretion. Conversely, others with a low secretion without obvious symptoms or gross conjunctival and corneal changes have presumably a diminished volume but a relatively higher concentration of some essential protective factor.

In spite of these difficulties in assessing the normal value for the filter-paper test, we can accept the figures quoted above as a good working guide, at any rate for conditions in Manchester, though they are higher than those found by Henderson and Prough (1950). But in assessing whether any particular patient has a mild or severe keratoconjunctivitis sicca, clinical signs and symptoms have been used entirely, mild cases being those with only minimal discomfort and little or no corneal involvement, severe cases having marked discomfort, obvious corneal changes and usually a strongly positive rose bengal test. On this basis, 26 of the patients were classified as severe and 24 as mild. 


\section{RELATIONSHIP TO ARTHRITIS}

Of the changes in other parts of the body often found in association with kerato-conjunctivitis sicca, arthritis has received considerable attention. Most workers consider arthritis to be part of the syndrome, though Henderson (1950) considers it to be an incidental finding only. To a certain degree his view is borne out by the fact that in the 26 severe cases in the present series, only six ( 23 per cent.) have rheumatoid arthritis, two (8 per cent.) osteo-arthritis, one Reiter's syndrome, and one palindromic arthritis. The mild cases of dry eye cannot be analysed in this way, as six of them were found on routine examination of arthritics. Nevertheless, the proportion of arthritics amongst patients with kerato-conjunctivitis sicca is significantly high; and conversely Stenstam (1947) found that one in ten of chronic arthritics has well marked kerato-conjunctivitis sicca, compared with one in 620 in an unselected group of ophthalmic patients (de Roetth, 1945). Henderson rather misinterprets Stenstam when he says that the latter considered it unlikely that arthritis was part of the ocular condition, for Stenstam looks on the kerato-conjunctivitis as a symptom of arthritis, saying:

the chronic arthritis type with a protracted morbid history and pronounced tendency towards shrivelling and destruction of the joints is the most definite keratoconjunctivitis sicca complication.

Sjögren (1948), in spite of Henderson's contrary view, still believes that both the arthritis and the kerato-conjunctivitis sicca syndrome are expressions of a chronic general infection.

Stenstam (1947) also made the remarkable observation that keratoconjunctivitis sicca was as common an accompaniment of arthritis in men as in women; my series of male arthritics, numbering only 28 , is too small to make comment on this point justifiable, but in the total of six male patients with dry eyes, the disease was of the mild type in five and severe in only one. This suggests that there must be some secondary, presumably endocrine, factor, which is operative mainly in women.

\section{Relationship to Dry Mouth}

The second and commonest accompaniment of dry eyes is a dry mouth. This was present in ten of the 26 severe cases, and conversely about half of all patients with xerostoma are said to have dry eyes (Faber, 1944). According to Rose (1950a) the main trouble in the mouth is not so much the dryness, to which the patient becomes accustomed, as the recurrent attacks of infection, particularly of the parotid gland, which becomes intermittently painful, enlarged, and tender. The involvement of the parotid is due to differences in its function from those of the other salivary glands, and under conditions of emotional stress causing dryness of the mouth even in normal people, the tongue "cleaves" to the roof of the mouth, not to the 
floor. The infection is commonly by the streptococcus viridans and is associated with an ectatic condition of the terminal ducts and acini as shown by sialography using a radio-opaque substance

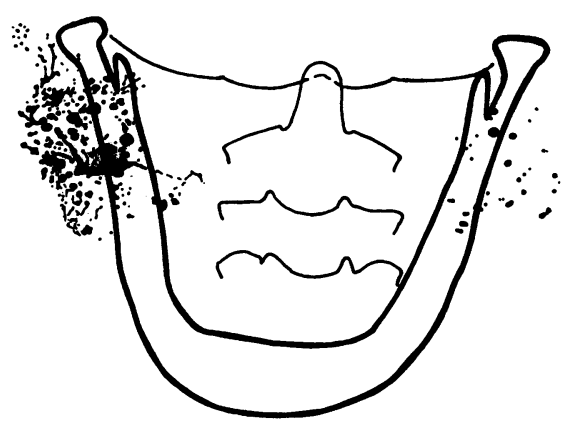

FIG. 1.-Line tracing of radiograph taken after sialography showing retention of radio-opaque substances in dilated terminal ducts and acini. (Neo-Hydriol fluid). (Fig. 1). Though the histological changes in the parotid and lacrimal glands are comparable (Sjögren, 1940), the clinical picture in the mouth differs from that usually seen in the eye for one rarely finds swollen, tender lacrimal glands in patients with dry eyes.

Case 1. Woman aged 48 attended Dr. W. Stirling's out-patient department in August, 1946, with a painful swelling of the right lacrimal gland. X-ray examination and Wassermann reaction were negative. The swelling was removed surgically in September, 1946, and in June, 1947, she developed kerato-conjunctivitis sicca. In February, 1948, Schirmer's test was right $6 \mathrm{~mm}$., and left $30 \mathrm{~mm}$. The rose-bengal test was strongly positive in the right eye. In February, 1949, she had some tenderness, and pain over the left lacrimal gland which settled down in ten days. In October, 1949, Schirmer's test was right $5 \mathrm{~mm}$., left $25 \mathrm{~mm}$.; and 12 months later right $3 \mathrm{~mm}$., left $20 \mathrm{~mm}$. Subsequent obliteration of the lacrimal canaliculi of the right eye by diathermy made her a little more comfortable for a short time, but had no influence on the result of the filter-paper test.

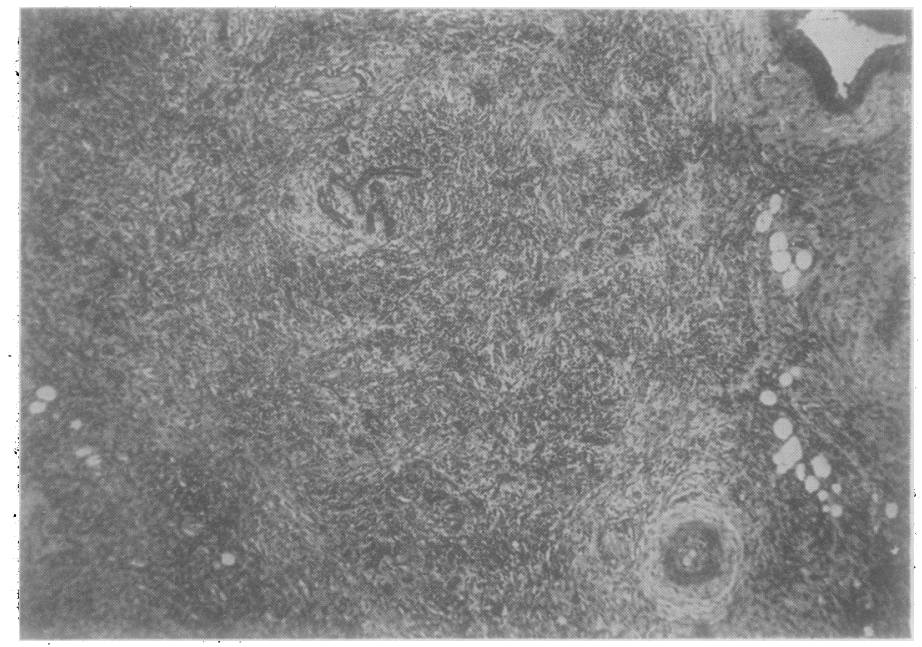

FIG. 2.-Case 1. Histological appearance of lacrimal gland. Haematoxylin and eosin. x 30 .

Pathological Report (Dr. J. L. S. Smith) of section of the right lacrimal gland.

The individual gland elements are widely separated by excess overgrowth of the stroma. Normal-looking acini are relatively scarce and the terminal units of the gland 


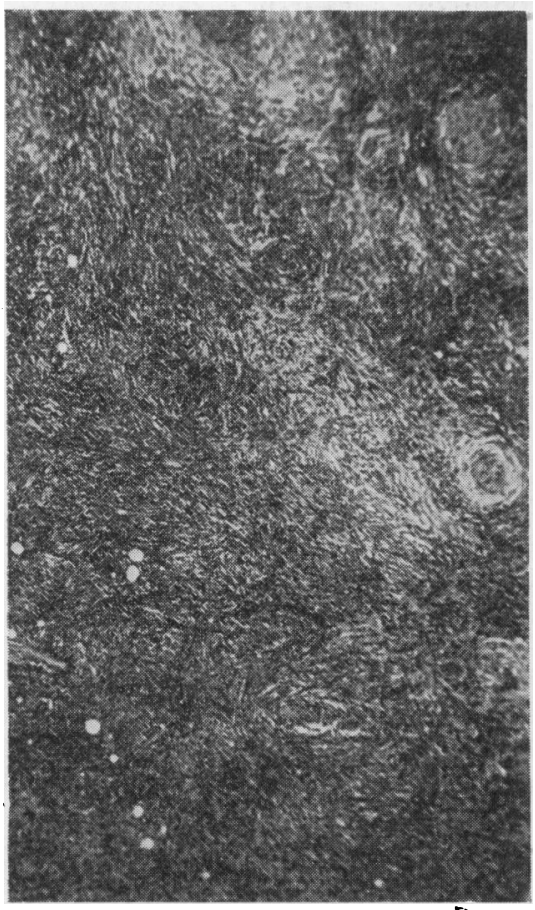

FIG. 3.-Case 1. Histological appearance of lacrimal gland showing more advanced changes. Haematoxylin and eosin. x 30.

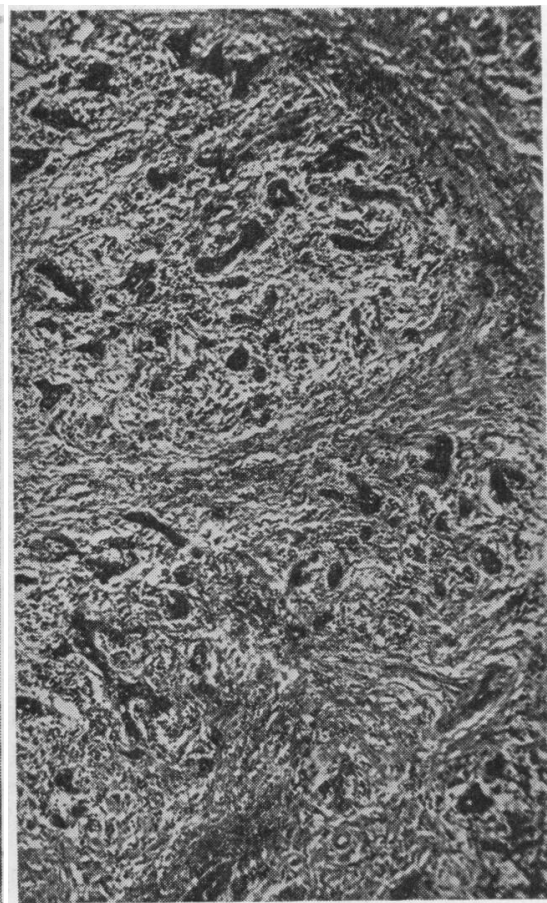

FIG. 4.-Case 2. Histological appearance of lacrimal gland. Haematoxylin and eosin. $\times 60$.

have mostly a ductule character with numerous eosinophilic plugs. In places they have largely disappeared or are unrecognizable. The stroma is richly lymphoid but there is considerable proliferation of the fixed tissue cells, especially around the vessels, where the round cells are often few and the deposition of fibrous tissue, frequently of hyaline nature, is widespread. Areas of lymphoid tissue have suffered fibrous replacement. Many of the vessels are occluded and many of the medium-sized vessels show a marked cellular endarteritis.

Nearly all the gland tissue is involved and a certain patchiness is evident as the processes have progressed further in some areas than in others. In large portions of the gland the changes are of a severe nature, suggesting that there may be considerable interference with lacrimal function (Figs 2 and 3).

Case 2. Man aged 61, had a swelling of the supero-lateral angle of the right orbit. The swelling was excised in September, 1950. There was no clinical evidence of kerato-conjunctivitis sicca, though the result of a filter-paper test is not known, and the patient was not seen by the author.

Pathological Report (Dr. J. L. S. Smith).

Sections show a portion of the lacrimal gland in which is seen a widespread separation of the individual glandular elements, together with some atrophy. Complete and partial casts formed from cellular debris lie in the ductules and acini. The connective tissue has proliferated considerably to produce an abundant stroma with new collagen and richly lymphoid areas.

This specimen comprises only a small portion of the gland and includes one lobule in which the changes are much less marked, and in which many normal acini can be seen. This suggests that the gland may still be functioning adequately. (Fig. 4). 
These histological changes, consisting largely of glandular degeneration with lymphoid infiltration and proliferation of connective tissue, are very similar to those described by Sjögren (1940) in cases of kerato-conjunctivitis sicca. Unfortunately, the nature of the pathological changes in the lacrimal glands in kerato-conjunctivitis sicca is a subject of dispute (Holm, 1949), and it is not, therefore, possible to be certain that these patients had an unusual type of the disease. Nevertheless, this possibility is supported by the following considerations :

(1) In Case 1, the left eye in which the lacrimal gland is still present, has shown a steady decrease in the amount of lacrimal secretion over a period of two years, though it is still within the normal range.

(2) The physiological changes in the lacrimal gland are always far in advance of the clinical changes in the conjunctiva or cornea (Sjögren, 1940).

(3) Removal of the lacrimal gland does not always result in kerato-conjunctivitis sicca (Duke-Elder, 1939), presumably because the accessory lacrimal glands are able to produce sufficient moisture. It is possible, then, that the development of keratoconjunctivitis sicca after extirpation of the lacrimal gland, as in Case 1, indicates that the accessory glands are also involved in the disease process. This could equally be the case in those patients whose dry eyes follow functional " removal " of the lacrimal gland by such things as a fractured skull (Coverdale, 1948), since it may well be that the accessory glands have the same innervation and consequently suffer the same changes as the main lacrimal gland.

If this possibility is accepted, then those obscure cases of Mikulicz' syndrome of enlarged parotid and lacrimal glands in which there is no suggestion of lymphatic leukaemia or sarcoidosis, may fall into the same group as kerato-conjunctivitis sicca. A similar suggestion hás been made recently by Ellmann and Weber (1949) and Holm (1949).

That one may in fact get sufficient increase in the size of a lacrimal gland to produce obstruction of the venous and lymphatic return of the lid, without the lacrimal gland being palpable, is suggested by the following case.

Case 3. Woman aged 62 attended Mr. O. M. Duthie's out-patient department in October, 1948, with a history of partial ptosis of the right upper lid for six months which was worse on waking in the morning and towards the end of the day. The extra-ocular muscles were normal and the action of the levator palpebrae superioris was present. She had no other neurological' signs or symptoms. The skin of the right lid looked very redundant, but there were no other obvious local changes. $X$-ray examination of the skull and Wassermann reaction were negative. Prostigmine had no effect on the ptosis, which, with no further treatment, practically disappeared within four months. In October, 1950, she attended again with six months' history of swelling of the lacrimal, parotid, submaxillary, and cervical glands. The lacrimal secretion was normal by Schirmer's test. She was referred to Professor Boyd, and a diagnosis of sarcoidosis was established by biopsy.

Presumably the ptosis in this case resulted from the first slight 
swelling of the gland, but cleared up as the vascular conditions adapted themselves to the new situation. That a similar picture may be seen in patients with kerato-conjunctivitis sicca is suggested by the two following cases.

Case 4. Woman aged 53 attended Dr. F. Janus's out-patient department in January, 1948, with a well-marked bilateral kerato-conjunctivitis sicca. Schirmer's test was right $5 \mathrm{~mm}$. and left $5 \mathrm{~mm}$. : the rose-bengal test was slightly positive in both eyes. Her mouth and tongue were sometimes sore, but not noticeably dry. She had osteoarthritis, complete achlorhydria, an atrophic skin, purpura, and a normal blood count, including platelet count. She also complained of ptosis (Fig. 5) which was much worse in the mornings; the whole face was heavy-looking, but there was no evidence of thyroid deficiency, the basal metabolic rate being within normal limits. The ptosis was not influenced by prostigmine. The achlorhydria was treated with hydrochloric acid with meals, and in view of a somewhat sub-normal dark-adaptation curve, Tab. vitamin A 24,000 units thrice daily was given. This had no effect on the signs and symptoms or on the dark-adaptation curve. The lacrimal canaliculi of the right eye were occluded by diathermy in August, 1949. She had a temporary remission of her ocular irritation for a few weeks, but subsequently relapsed to her former condition, though the puncta still remained occluded.

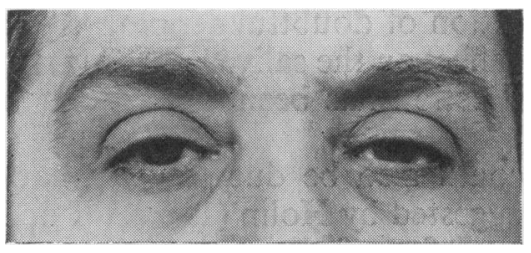

FIG. 5.-Case 4. General appearance of face showing slight bilateral ptosis.

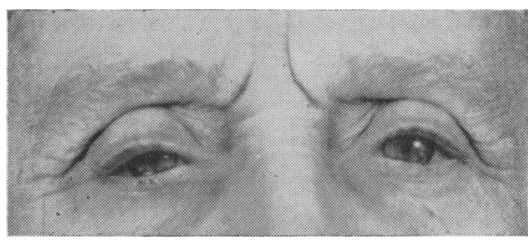

FIG. 6.-Case 5. General appearance of face showing bilateral ptosis, more marked in the right eye.

Case 5. Woman aged 63 had been attending the out-patient department since 1942 with irritable eyes. I first saw her in March, 1948, when both eyes showed a typical stain with rose bengal; Schirmer's test was right $12 \mathrm{~mm}$. and left $14 \mathrm{~mm}$. She had also a dry mouth with slight swelling of the parotids. She complained of drooping of the lids especially in the mornings (Fig. 6). The ptosis did not respond to prostigmine. She remained reasonably comfortable on treatment with gutt. gelatine in Locke's solution.

In these cases the increased weight of the lids producing ptosis may have been caused not only by oedema due to obstruction, but also by swelling of the accessory lacrimal glands which would also be implicated in this disease. It may be of some significance that all three patients with ptosis stated definitely that it was worse on waking in the morning, the lack for a few hours of the pumping action of the lid movements on the vascular circulation having presumably led to greater congestion. 


\section{Conjunctival Changes}

A further pathological change of great interest is the sub-epithelial oedema of the conjunctiva (Sjögren, 1940). One may ask why it occurs in kerato-conjunctivitis sicca, but not in other forms of drying. Possibly it is in the nature of an axon reflex with liberation of histamine-like substances as a result of the greater liability to trauma. It may then occur in kerato-conjunctivitis sicca and not in other forms of exposure, because the former is not merely a local disease of the lacrimal glands but a general disease of the conjunctiva, which involves the vessels in such a way as to alter their reactions to such reflexes. This theory is supported by the remarkable effect of bilateral section of the vertebral nerves and bilateral resection of a portion of the suprastellate segment of the cervical chain performed in a case reported by Leriche (1947) for its effect on the circulatory system of the face. Within six months the mouth was moist again and the patient could shed tears. This is obviously a line of treatment that needs following up, though cases of kerato-conjunctivitis sicca do show a fairly marked tendency to spontaneous remissions that makes assessment of therapy somewhat difficult, and Leriche's patient had also a somewhat atypical facial condition of doubtful aetiology. In addition, Rose (1950b) has found no effect on the salivary secretion in some of his patients on whom an injection has been carried out to paralyse the stellate ganglion.

The abnormality in the vessels could also be due to a primary change in their nerve supply, as is suggested by Holm (1949). I find his arguments unconvincing, however, for of the two patients he cites as examples of kerato-conjunctivitis sicca symptoms with a neurological pathogenesis, one may not have been a case of keratoconjunctivitis sicca at all (since mere corneal staining by rose bengal cannot be considered to be diagnostic), and the other patient had a long-standing facial paralysis and inability to close the eye, a condition which could conceivably lead to blocking of the ducts or destruction of the secretory glands by chronic inflammation. In addition, it is not surprising that patients with a deficient lacrimal secretion under ordinary conditions can nevertheless shed tears when they receive some more unusual and powerful stimulus; there is no criterion by which we can judge whether or not the amount of fiuid produced when a patient sheds tears is or is not normal.

\section{TREATMENT}

The treatment of the condition may be directed towards the supposed causes, or may be merely symptomatic.

Whatever the common factor between the arthritis and mild dryness of the eyes may be, no adequate treatment of it can be 
envisaged, unless cortisone proves useful. In any case, the secondary possibly endocrine, factor, which is responsible for the more severe manifestations, is probably of more clinical importance. Though many observers have reported considerable improvement with various forms of endocrine treatment with both male and female hormones, my own feeling is that any action of these compounds may well have resulted indirectly from an improvement in the patient's general condition.

Vitamin A has also been used, possibly because it is known that xerosis produces drying, and possibly because the later stages of kerato-conjunctivitis sicca show histological conjunctival changes somewhat similar to those in xerosis. The major conjunctival change in kerato-conjunctivitis sicca, however, is the sub-epithelial oedema, which is not present in other forms of drying. Certainly I have found no improvement in the clinical course or in the darkadaptation curves of four patients to whom I have given vitamin A.

A further method of treatment is the attempt to stimulate the secretory fibres of the lachrymal gland. I have had no results from pilocarpine given parenterally, or from prostigmine (which failed to affect either the dryness or the ptosis in Cases 4 and 5). Local pilocarpine and eserine certainly have no effect which outweighs their other disadvantages.

We come finally, then, to local treatment; in his cases of dry mouth, Rose (1950b) uses local injections of penicillin into the parotid duct, parenteral penicillin being ineffective. But in the lachrymal gland there are a dozen or so small ducts, which make such local therapy impossible, and parenteral penicillin is equally ineffective. $X$-ray treatment to the parotid may help the secondary infection, and the swellings in Mikulicz' disease are usually radiosensitive (Ellman and Weber, 1948), but such treatment has no effect on the dryness, and indeed one might expect that the consequent increase in fibrosis might make the secretions even more deficient.

The most promising local treatment is, of course, diathermy to the canaliculi (Beetham, 1935). This often gives immediate and striking relief, but Henderson (1950) has shown, by a follow-up study, that only one patient in three is really benefited, and that some are made worse by having a troublesome pool of tears at the inner canthus, as in the following example:

Case 6. Woman aged 62 first attended Mr. O. M. Duthie's out-patient department in November, 1948. Schirmer's test was right $8 \mathrm{~mm}$., left $6 \mathrm{~mm}$., and the rose-bengal stain was positive in each eye. Conservative treatment with gutt. gelatine in Locke's solution produced little subjective improvement, Schirmer's test in May, 1948, being right $9 \mathrm{~mm}$., left $5 \mathrm{~mm}$. Diathermy of the left canaliculi was then carried out, after which the patient complained of a watering eye. This condition persisted, and in November, 1950, Schirmer's test was right $9 \mathrm{~mm}$., left $24 \mathrm{~mm}$. In view of this, the lower canaliculus was re-opened, an operation which was quite easy; the canaliculi 
tended to close again, however, and a small Weber operation was performed, after which it was possible to keep the passages open by occasional sac wash-outs. The watering was subjectively much improved, but Schirmer's test was still $24 \mathrm{~mm}$. in the left eye and in December, 1950, it was $24 \mathrm{~mm}$. in the right eye also.

Apart from the poor result from the diathermy of the canaliculi, the interesting point in this patient is the marked improvement in the lacrimal secretion of the right, unoperated eye, after repeated surgical procedures in the region of the left lacrimal passages. This suggests that the good results of diathermy to the canaliculi may be due not so much to blockage of the canaliculi as to the reflex stimulation of lacrimal-gland secretion. That there may indeed be such a reflex is suggested by Wolff (1948), who argues from the diminution in tear secretion that follows extirpation of the lacrimal sac. The effect of diathermy is explained thus much more adequately than by a theory of simple conservation of such secretion as is present; in the normal eye, a large proportion of the tears is lost by evaporation, and in patients with diminished secretion it would be expected, assuming that the rate of evaporation was the same as in the normal individual, that by far the greater proportion would be lost in this way. The conservation of the small volume of tears passing down the lacrimal passages by closure of the canaliculi would therefore have very little effect on the total amount of moisture in the eye. In Case 1, in whom the lacrimal gland had been removed so that diathermy of the canaliculi could not initiate a reflex increase in lacrimal secretion and the only effect of the operation could be conservation of such tears as were present, it is noteworthy that no objective and only slight subjective improvement was produced. Possibly the relatively prolonged effect of the diathermy in the average patient is due to the removal of some secondary blockage of the lacrimal ducts by the suddenly increased lacrimal secretion, which has much the same effect as repeated local wash-outs of the infected parotid gland through its ducts. In time, however, the rate of secretion falls away, epithelial debris and secretion accumulate in the ducts again, and the dryness of the eye returns, as in Case 4.

In general, I do not use diathermy except in those cases where conservative treatment with some form of artificial tears has failed to produce sufficient amelioration of the signs and symptoms.

\section{SUMMARY}

(1) Kerato-conjunctivitis sicca is a disease due to the combination of more than one factor, the first causing the association of a mild dry eye with arthritis, in both male and female, and the second, possibly endocrine, causing the severe manifestations in the female. 
(2) The same type of reaction may occur as a more acute and patchy process, causing a swelling of the lacrimal glands, linking the condition with the idiopathic type of Mikulicz' disease.

(3) The lacrimal swelling may cause ptosis, worse on waking in the morning.

(4) The disease process affects also the accessory lacrimal glands, and if the main gland is removed for any reason, kerato-conjunctivitis sicca follows only in those patients whose accessory glands have also been involved.

(5) This generalized process may have a vascular basis, thus accounting for the peculiar oedematous nature of the conjunctival changes, which result from axon reflexes in damaged vessels.

(6) It is preferable to start treatment with conservative measures and to use diathermy of the canaliculi only when the subsequent course of the disease justifies it.

(7) The effect of diathermy is due less to the conservation of the tears already present than to a reflex stimulation of the lacrimal secretion.

I wish to thank Dr. J. L. S. Smith for the pathological reports and many valuable suggestions, Mr. S. S. Rose for his great help and for the radiograph on which Fig. 1 is based, the Department of Medical Photography of the Manchester Royal Infirmary for Figs 1, 5, and 6, and the Surgeons of the Manchester Royal Eye Hospital for their willing co-operation in allowing me to see their patients.

\section{REFERENCES}

Beetham, W. P. (1935). Trans. Amer. ophthal. Soc., 33, 413.

COVERDALE, H. (1948). British Journal of Ophthalmology, 32, 669.

DE Roetth, A. (1945). J. Lancet, 65, 423. Quoted by Henderson (1950).

DUKE-ELDER, W.S. (1937). "Text-book of Ophthalmology", vol. 2, p. 1753. Kimpton, London.

Ellman, P., and Weber, F. Parkes (1949). Brit. med. J., 1, 304.

FABER, M. (1944). Acta med. scand., 118, 452.

Henderson, J. W. (1950). Amer. J. Ophthal., 33, 197.

and Prough, W. A. (1950). Arch. Ophthal., Chicago, 43, 224.

Holm, S. (1949). Acta Ophthal. Kbh., Suppl. 33.

KLEIN, M. (1949). British Journal of Ophthalmology, 33, 387.

LERICHE, R. (1947). Pr. méd., 55, 77.

RIDLEY, F. (1948). Quoted by M. Klein (1949).

Rose, S. S. (1950a). Brit. J. Surg. In the press. (1950b). Personal communication.

SCHIRMER, O. (1903). Graefes Arch. Ophthal., 56, 197.

SJögren, H. (1933-1940) See F. Ridley and A. Sorsby, " Modern Trends in Ophthalmology " (1940), chap. 38. Butterworth, London. (1948). Acta med. scand., 130, 484.

StenstaM, T. (1947). Ibid., 127, 130.

WOLF, E. (1948). "The Anatomy of the Eye and Orbit", 3rd ed., p. 189. Lewis, London. 